INTERNATIONAL JOURNAL OF MULTIDISCIPLINARY RESEARCH AND ANALYSis

ISSN(print): 2643-9840, ISSN(online): 2643-9875

Volume 04 Issue 12 December 2021

DOI: 10.47191/ijmra/v4-i12-31, Impact Factor: 6.072

Page No.- 1996-2001

\title{
Comparative Study of Abnormalities of the Spermocytogram between Manual Techniques versus Automated SCA (Sperm Class Analyzer)
}

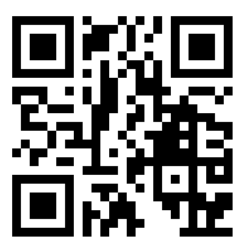

Zahra Ait Yachou ${ }^{1}$, Amina Benzaouiya ${ }^{1}$, Chaymaa Kabir-Idrissi Bouchra El Khalfi ${ }^{2}$, Mustafa Zakaria $^{3}$, Sabri yassir ${ }^{1}$, Mohammed Zarqoui ${ }^{4}$, Abdelaziz Soukri ${ }^{2}$, Achraf Zakaria ${ }^{1}$, Modou M. Mbaye, ${ }^{1,2,3}$, Noureddine Louanjli ${ }^{5}$

${ }^{1}$ Laboratory of Medical Analyses, Reproductive Biology, Labomac, Casablanca, Morocco

${ }^{2}$ Laboratory of Physiopathology, Genetics Molecular and Biotechnology (PGMB), Faculty of Sciences Ain Chock, Research Center Health and Biotechnology, University Hassan II of Casablanca, Morocco

${ }^{3}$ Association for Scientific Research of the IRIFIV-AISRG Group, Consultant in IRIFIV Fertility Center, IVF laboratory, Casablanca, Morocco

${ }^{4}$ GYN OBT IRIFIV Center, and IRIFIV-AISRG Scientific Research Group

${ }^{5}$ Labomac Clinical Analysis Laboratory, IRIFIV Fertility Center, AFC Fertility Center, Casablanca, Morocco

ABSTRACT: It is already well established that the percentage of normal sperm and specific sperm abnormalities has diagnostic value in vivo. The spermocytogram is an important part of the analysis of human semen, this kind of analysis, simple at first sight, brings real difficulties because the results from one laboratory to another are very relatively reliable. When analyzing abnormal sperm morphology, the observer has to estimate the respective size of the different spermatozoa or their components, the length of all the spermatozoa (too small or too big) and to identify the shape (sperm with a coiled flagellum, or no flagellum as an example). The objective of our work is first of all to make a comparative study of the results of the morphological evaluation of 50 semen samples between the manual technique versus the automated one (SCA) in order to find a possible correlation between these two techniques. According to the analytical stage of the manual and automatic results, the two latter led us to obtain significantly different percentages.

KEY WORDS: spermatozoa, spermocytogram, SCA.

\section{INTRODUCTION}

Infertility is defined as the inability to achieve pregnancy after 12 months or more of regular unprotected sex. According to the World Health Organization [1] , it affects approximately $15 \%$ of couples worldwide, or 48.5 million couples. As such, it is a real public health problem. Male infertility alone accounts for 20-30\% of infertility cases [2].

The causes of male infertility are many and varied. We can cite among others: the problems of sperm dejection, the absence or low sperm count, reduced mobility of sperm, and morphology [3].

Sperm morphology is a very important, crucial, and preponderant parameter for the success or failure of in-vitro fertilization. In this regard, several clinical researches have shown that, unlike sperm count and motility, sperm morphology is one of the most stable parameters for the evaluation of sperm fertility. In this sense, it allows us to examine the health of the testicle and serves as a predictor of possible male fertility.

Moreover, the latest recommendations of the WHO 2010 guide have emphasized the need to standardize these examinations [4]. To meet this demand for consistency, automated spermiology machines have advanced to the point where they are now a viable alternative to manual sperm analysis, allowing a combination of high reliability and low variability [5].

The aim of this research paper is to make a comparative study of the results of the morphological evaluation of 50 semen samples between the manual technique versus the automated (SCA) in order to find a possible correlation between these two techniques. 
Comparative Study of Abnormalities of the Spermocytogram between Manual Techniques versus Automated SCA (Sperm Class Analyzer)

\section{MATERIAL AND METHODS}

\subsection{Collection of the samples}

The collection of samples was carried out at the laboratory of medical analysis, biology of reproduction, LABOMAC, Casablanca, Morocco. The study involved 50 samples from male volunteers. Informed consent was obtained from all men included in this study before their samples were used. Samples were collected by masturbation after 3 to 4 days of sexual abstinence in sterile, labeled vials. Once collected, the samples were left at $37^{\circ} \mathrm{C}$ for liquefaction for $30-60 \mathrm{~min}$. We checked at $10 \mathrm{~min}$ intervals to ensure that liquefaction was complete.

Microscopic analysis was performed according to World Health Organization [1]. standards and guidelines.

\subsection{Processing of the samples}

After one hour of semen production, a routine spermogram was performed to determine sperm count, motility, vitality, using the $20 \mu \mathrm{l}$ Makler counting chamber. The pretreatment of the samples, we had to realize for each sample two smears, dried, fixed and stained before the evaluation of the morphology are by the manual and automatic technique (figure 1).

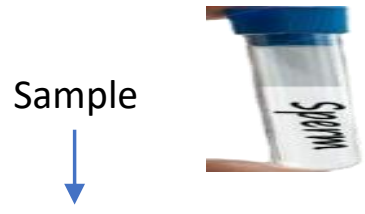

Liquefaction à $37^{\circ} \mathrm{C}$<smiles>[C]1C=C1</smiles>

\section{$0.5 \mathrm{ml}$ of sample $+1 \mathrm{ml}$ of Earl)}

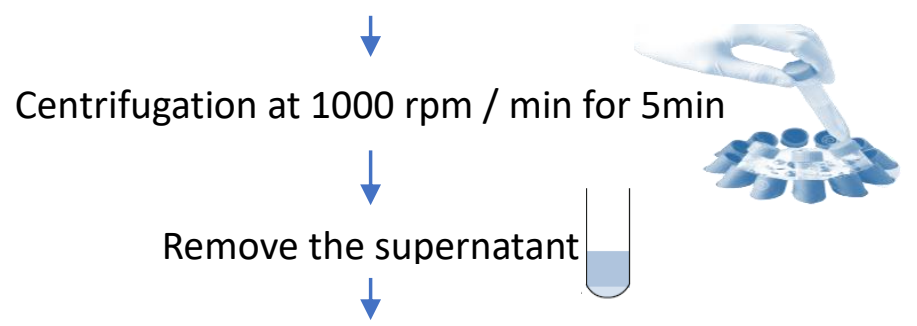

Make a smear with the pellet let it dry at room temperature.

Fixation with ethanol for $30 \mathrm{sec}$

let dry at room temperature.

Cover the slide with hematoxylin for $5 \mathrm{~min}$, wash

Immerse slide in Shorr reagent for $3 \mathrm{~min}$, wash<smiles>[CH]1[CH]C1</smiles>

Let it dry
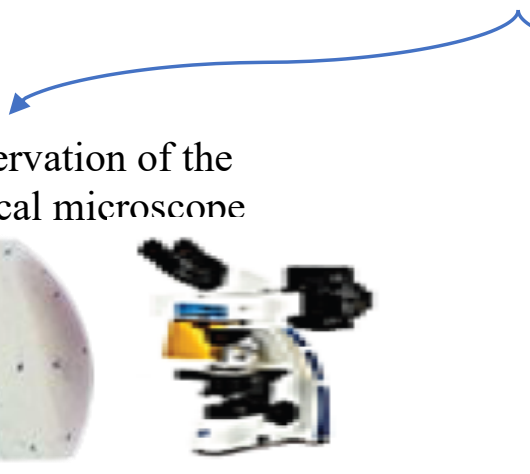

Manual observation of the

slides by optical microscone

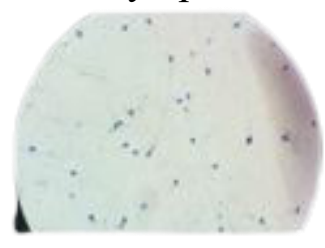

Automatic analysis of

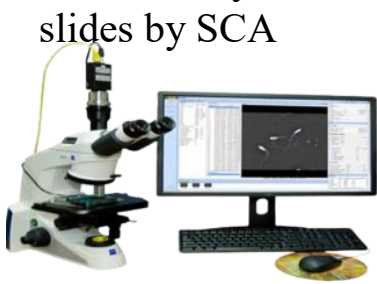

Figure 1: Sample processing treatment methodology 
Comparative Study of Abnormalities of the Spermocytogram between Manual Techniques versus Automated SCA (Sperm Class Analyzer)

\subsection{Evaluation of morphology}

The manual evaluation of morphology was done by immersion in white light at $x 100$ magnification. On the other hand, the automated analysis was performed using the Sperm Class Analyzer with the CASA (Computer-Assisted Sperm Analyzer) system.

\subsection{Statistical analyzes}

The data obtained during our experiment were the subject of a statistical study. The results of EO supplementation on the physiological parameters (motility, vitality, mean travel speed (VAP) of human sperm were carried out by Student's $t$ test ( $t$ test). All the graphs and histograms represented in this article were produced using the software: GraphPadPrism7.

\section{RESULT}

\subsection{Normal spermatozoa}

Analysis of our normal sperm results showed a weakly significant difference between automated SCA analysis and manual analysis ( $p<0.05$ ) (Figure 2).

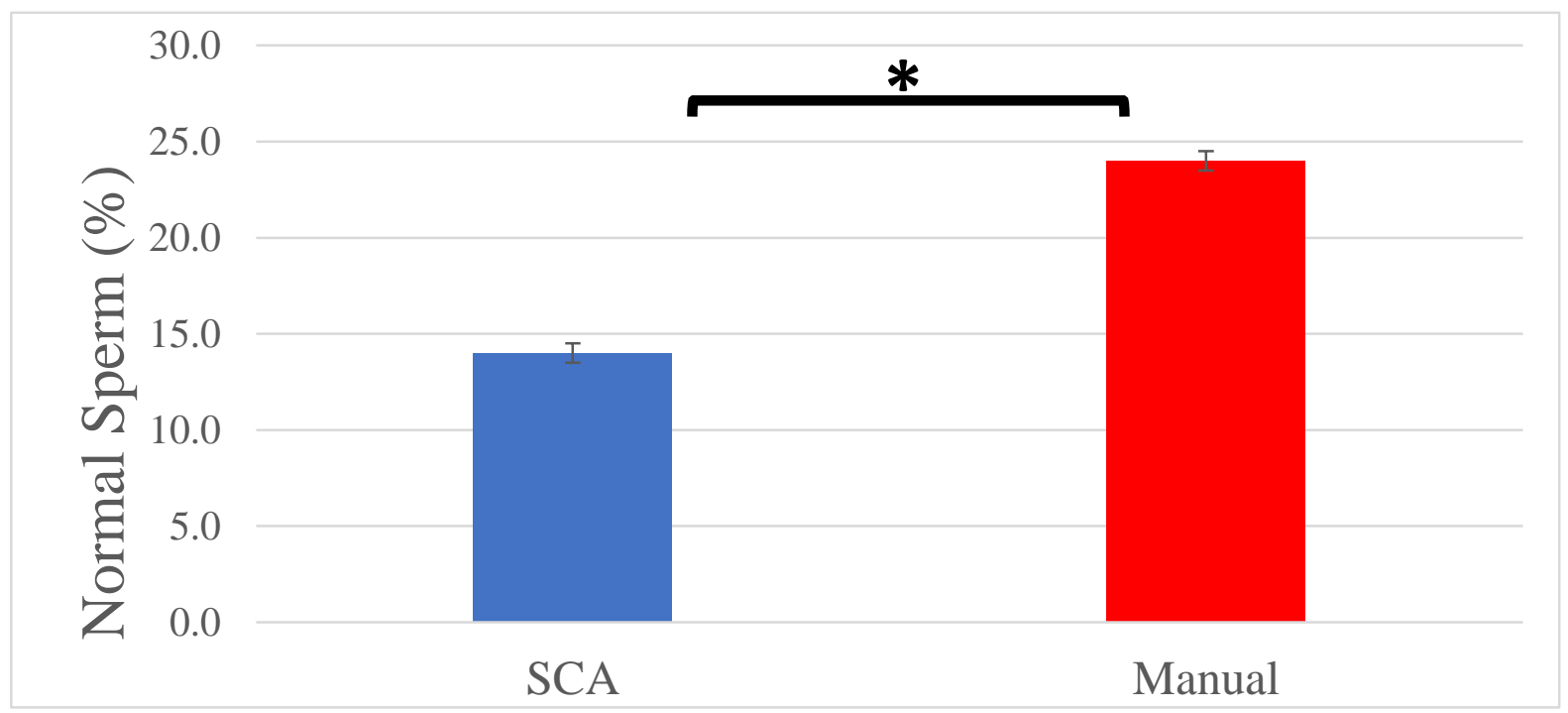

Figure 2 : Differences in manual and automatic SCA values of normal spermatozoa.

\subsection{Abnormal spermatozoa}

The morphological results of the abnormal sperm revealed a weakly significant difference between the automatic and manual SCA technique $(p<0.05)$ (Figure 3$)$.

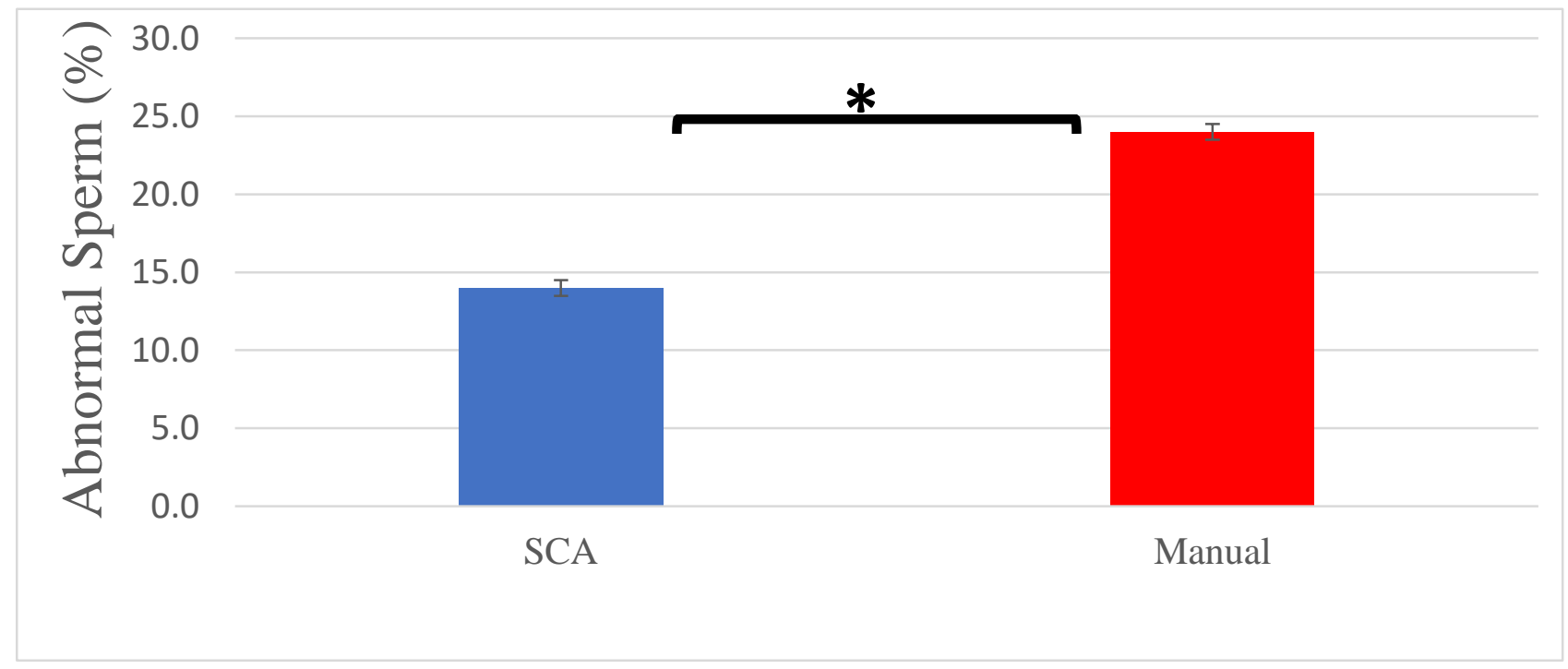

Figure 3 : Differences in manual and automatic SCA values of spermatozoa Abnormal. 
Comparative Study of Abnormalities of the Spermocytogram between Manual Techniques versus Automated SCA (Sperm Class Analyzer)

\subsection{Head Abnormal}

Analysis of morphology results for sperm head abnormalities revealed a difference between the automatic SCA technique and manual $(p<0.05)$ (figure 4).

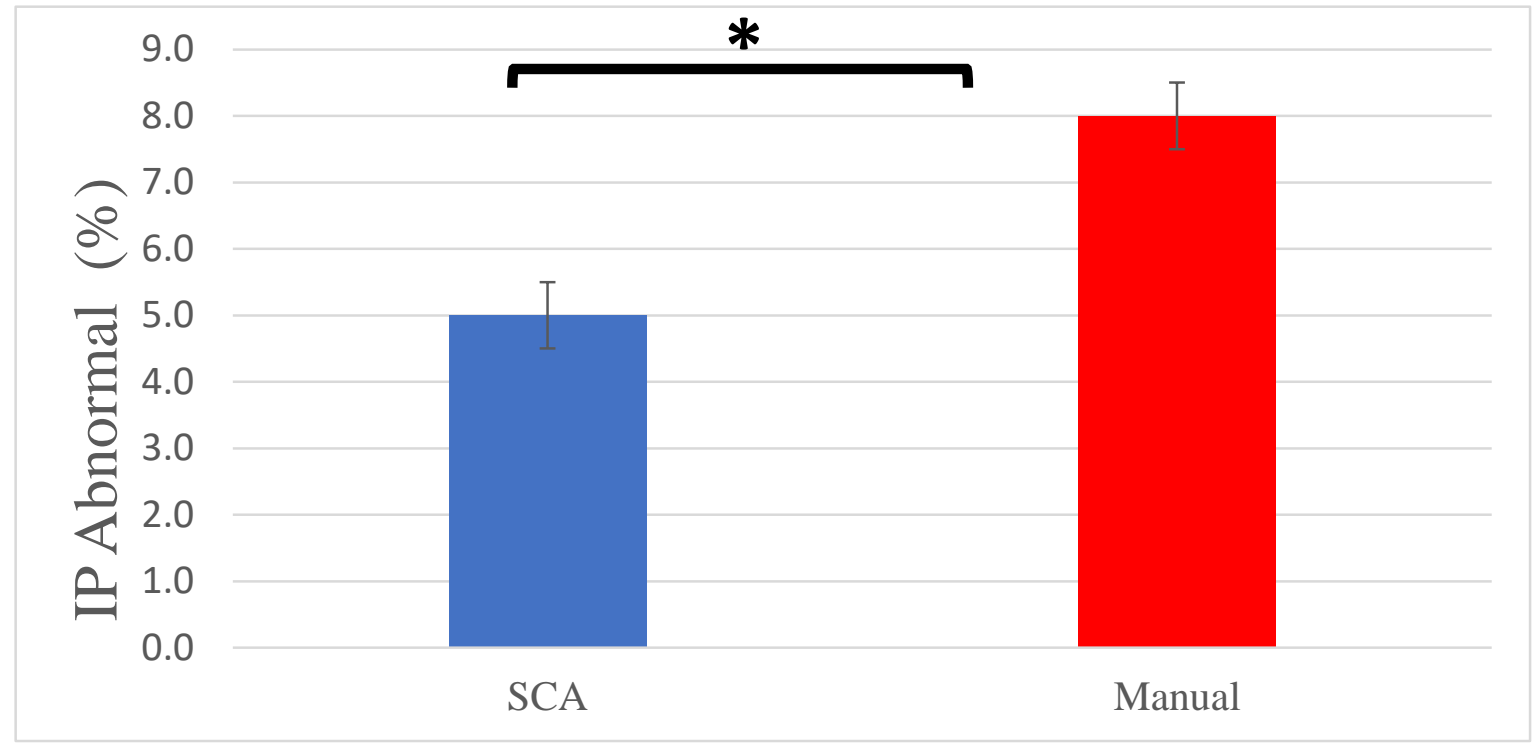

Figure 4: Differences in manual and automatic SCA values of sperm intermediate piece anomalies.

\subsection{Anomalies of the intermediate piece}

Analysis of our results for abnormalities of the sperm intermediate part showed a weakly significant difference between the automated analysis by ACS and the manual analysis $(P<0.05)$ (Figure 5).

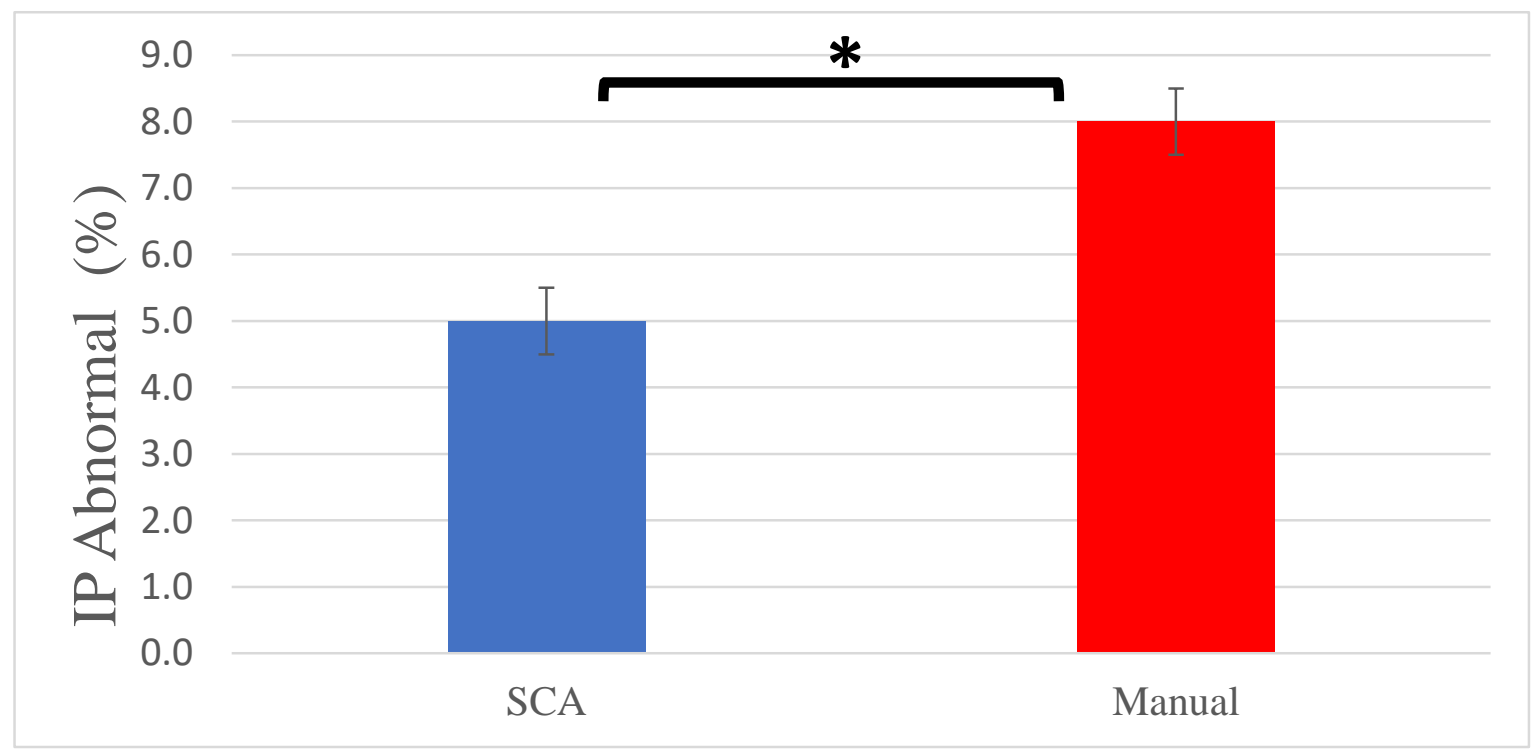

Figure 5 : Differences in manual and automatic SCA values of sperm intermediate piece anomalies.

\subsection{Flagellum Abnormal:}

Analysis of the morphology results for flagellar abnormalities revealed a significant difference between the automatic SCA and manual $(p<0.01)$ techniques (Figure 6$)$. 
Comparative Study of Abnormalities of the Spermocytogram between Manual Techniques versus Automated SCA (Sperm Class Analyzer)

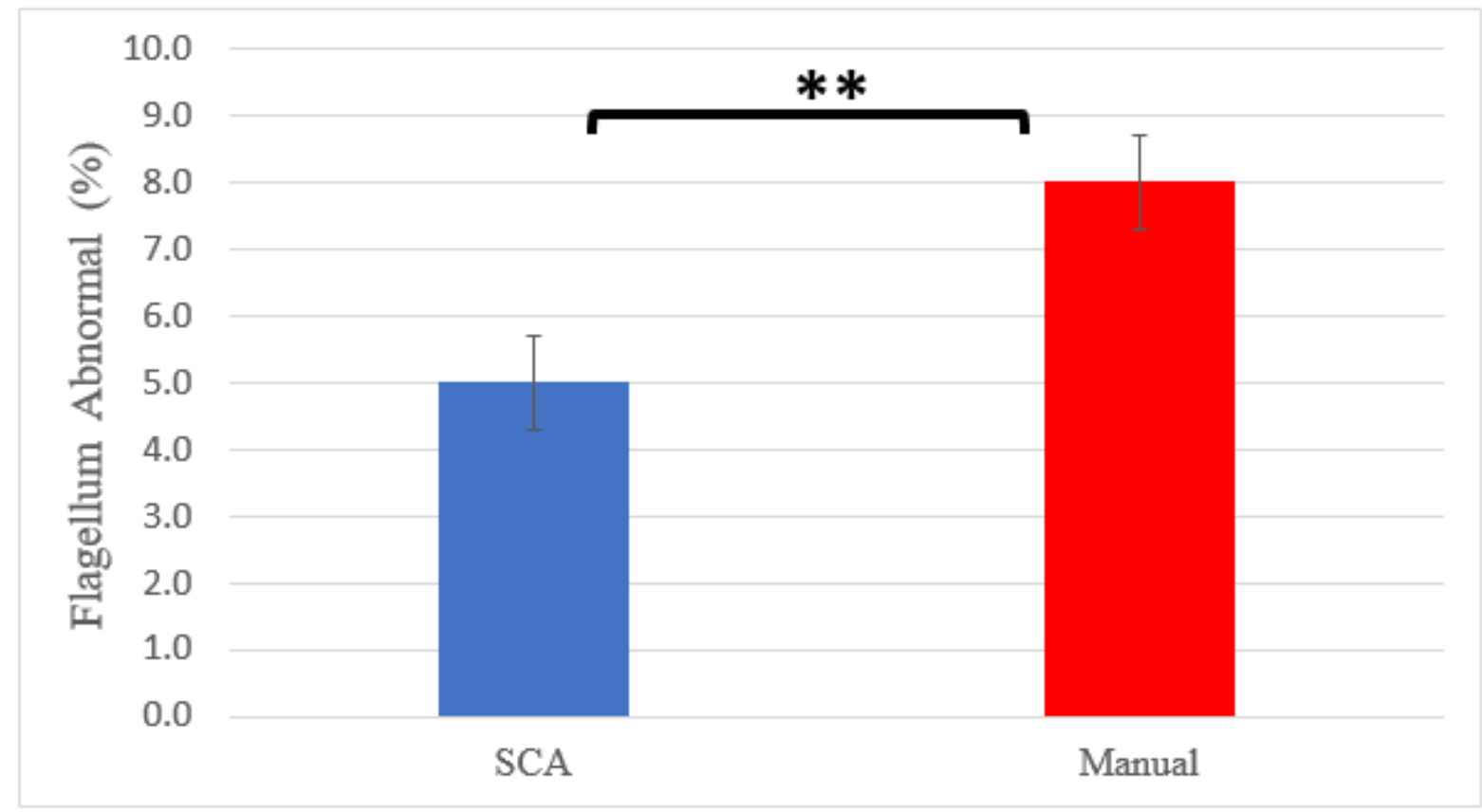

Figure 6: Differences in manual and automatic SCA values of sperm flagellum anomalies.

\subsection{Cytoplasmic remains}

The analysis of our spermocytogram results concerning the cytoplasmic remainder showed a significant difference between the automated analysis by ACS and the manual analysis ( $p<0.01)$ (figure 7 ).

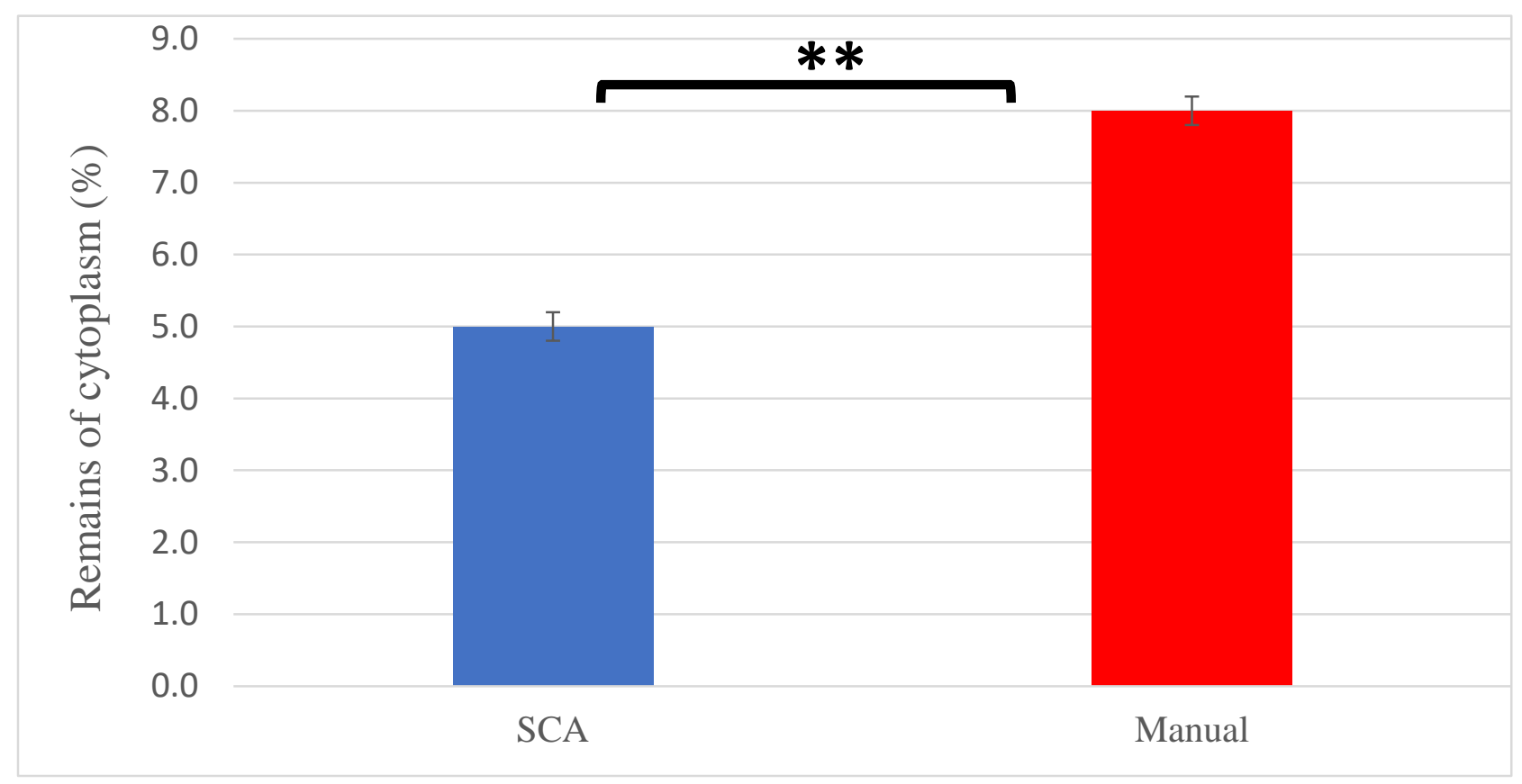

Figure 7: Differences in manual and automatic SCA values of cytoplasmic remains.

\section{DISCUSSION}

The objective of our work is first of all to make a comparative study of the results of the morphological evaluation of 50 semen samples between the manual technique versus the automated one (SCA) in order to find a possible correlation between these two techniques. Analysis of our normal sperm results showed a weakly significant difference between automated CSA morphology analysis and manual analysis ( $p<0.05$ ) (figure 2). Analysis of abnormal sperm revealed a significantly small difference between automatic SCA and manual technique $(P<0.05)$ (Figure 3). Regarding the abnormalities of the sperm head revealed a weakly significant difference between the automatic technique by SCA and manual (Figure 4). The non-difference between significant sperm intermediate part abnormalities from automated SCA manipulation and manual analysis was weakly significant (6\%) (Figure 


\section{Comparative Study of Abnormalities of the Spermocytogram between Manual Techniques versus Automated SCA (Sperm Class Analyzer)}

5). Analysis of the morphological results of flagellar abnormalities and cytoplasmic rest revealed a significant difference between the automatic and manual ACS technique $(P<0.01)$ (figure $6 ; 7)$.

In contrast to the work [6] which was conducted on a prospective study comparing semen analysis results; normal concentration, count, motility, and morphology obtained manually and by two automated systems, SQA-V GOLD (Sperm Quality Analyzer, Medical Electronic Systems, Los Angeles, CA, USA) and on computer-assisted semen analysis (CASA, CEROS Sperm Analyzer version 12.2 L, Hamilton Thorne, Beverly, MA, USA), following the WHO 5th edition recommendations. And it resulted in significantly different results for normal morphology reported by the SQA-V GOLD and CASA to the manual results according to the statistical analysis [7]. The results of our study concerning morphology, for sperm head abnormalities revealed a minimal difference between the automatic SCA technique and manual (Figure 3). Our results are in perfect agreement with those of the study conducted on although the average difference between the two methods was small and not significant [8].

\section{CONCLUSION}

Automated semen analyzers can be used for routine semen analysis, providing rapid clinically acceptable results with better accuracy and a positive impact on laboratory standardization. Giving better correlations between manual and automated method. Automated analysis of sperm morphology allows an objective measurement of the different morphometric parameters of the spermatozoon. The study of the sperm head seems to be the most accessible to automated analysis and should be able to provide selective elements in the evaluation of fertility. On the other hand, automated analysis of sperm abnormalities seems illusory with the current systems, the intermediate piece and the flagellum being small structures with little color and difficult to identify by the computer [9]. Hence the interest of manual spermocytogram analysis. All this led us to conclude that the spermocytogram can be accurately evaluated both manually and by automatic methods which allows to reduce intra and inter laboratory variations, even if the manual method requires additional time and an experienced technician compared to the automatic method.

\section{REFERENCES}

1) World Health Organisation. (2010). Examination and processing of human semen.

2) Agarwal, A., Mulgund, A., Hamada, A., \& Chyatte, M. R. (2015). A unique view on male infertility around the globe. Reproductive biology and endocrinology, 13(1), 1-9.

3) Khelifa, M. B. (2013). Identification et caractérisation de gènes impliqués dans l'infertilité masculine (Doctoral dissertation, Université de Grenoble; UNIVERSITE DE TUNIS EL MANAR).

4) Pons-Rejraji, H., Kaspraza, J., Harzallah, I., Vorilhon, S., Bouche, C., Pereira, B., \& Brugnon, F. (2020). Quelle est la place de l'automatisation du spermogramme-spermocytogramme dans un laboratoire de Biologie de la Reproduction: performance et validation de méthode de l'automate du SQA-Vision ${ }^{\circledR}$. In FFER.

5) Lammers J, Splingart C, Reignier A, Catteau A, Barrière P, Fréour T. Quelle place pour le spermogramme automatisé en 2016.

6) Lammers, J., Splingart, C., Barrière, P., Jean, M., \& Fréour, T. (2014). Double-blind prospective study comparing two automated sperm analyzers versus manual semen assessment. Journal of assisted reproduction and genetics, 31(1), 35-43.

7) Frikh, M., Benaissa, M., Kasouati, J., Benlahlou, Y., Chokairi, O., Barkiyou, M., Chadli, M., Maleb, A., \& Elouennass, M. (2021). Prévalence de l'infertilité masculine dans un hôpital universitaire au Maroc.

8) Moreau J, Reignier A, Leandri R, Parinaud J, Fréour T, Gatimel N. Spermogramme et spermocytogramme manuels et automatisés-Test de migration-survie. Rev Francoph Lab. juillet 2018;2018(504):28-35

9) Lannou, D. (1997). L'analyse automatisée de la morphologie du spermatozoïde. Basic and Clinical Andrology, 4(7), 427-432. 